\title{
Image-containing InGaN LEDs for pattern-transfer applications
}

\author{
D. Massoubre, B. Guilhabert, Z. Gong, I. M. Watson, E. Gu and M. D. Dawson \\ Institute of Photonics, University of Strathclyde, Glasgow, Scotland, UK, G4 ONW \\ Email addresses: david.massoubre@strath.ac.uk; benoit.guilhabert@strath.ac.uk
}

\begin{abstract}
We report a simple process which enables the fabrication of a single light-emitting diode with spatially patterned emission. The patterning is based on the selective passivation of the p-doped $\mathrm{GaN}$ with a $\mathrm{CHF}_{3}$ plasma treatment.
\end{abstract}

Introduction: The projection of micro-size images with complex features is of great interest for many applications including micro-displays, mask-less lithography and lab-on-chip sensors. For such applications, it is important that the projected image combines high brightness and highly uniform emission. To create such microimages, gallium nitride (GaN)-based light-emitting diode (LED) arrays designed in matrix-addressed configurations have already been demonstrated, and have shown good performance [1]. This is attributable to the combined advantages of the GaN-based LEDs (compactness, high output power, low power consumption, visible wavelength emission) [2] and of the matrix-addressed scheme (scalability, high resolution) [1]. Unfortunately, such configurations require numerous and complex processing steps [3]. Furthermore, the resolution is limited by the pixel pitch, typically on the order of few tens of micrometers $[1,3]$. These typical pitches are relatively large compared to the total size of the micro-image to be displayed, leading to deleterious pixelation. In order to overcome these problems, we propose a new approach to create micro-images from GaNbased LEDs with much higher resolution and a much simpler process flow than with the standard matrixaddressed scheme. This new approach is based on the combined use of a $\mathrm{CHF}_{3}$ plasma treatment and a standard photoresist (PR) mask to dramatically increase the resistivity of the p-type GaN layer in selected area [4]. It allows permanent spatial modulation of the light emission across the device, and the display and transfer of arbitrarily complex images.

Design and fabrication of the devices: In order to demonstrate this new approach, standard broad-area ('conventional chip') GaN-based LEDs were fabricated from commercially available p-i-n LED wafers grown on a c-plane sapphire wafer, and with peak emission wavelengths either at $450 \mathrm{~nm}$ or $370 \mathrm{~nm}$. First, the wafers were cleaved and cleaned in successive baths of acetone, methanol, isopropanol and deionised water, then dried with a nitrogen flow. The samples were next soaked $3 \mathrm{~min}$ in an $18 \%$ aqueous $\mathrm{HCl}$ solution to remove the native oxide, and then they were rinsed $3 \mathrm{~min}$ in deionised water. This was immediately followed by the deposition with an electron beam evaporator of a bilayer of $\mathrm{Ni} / \mathrm{Au}(6 \mathrm{~nm} / 6 \mathrm{~nm})$. Next, a thin photoresist layer was spin-coated on each sample and patterned by standard photolithography to define the mesa for each broad-area LED, and the mesas were then created by inductively coupled plasma dry etching down to the n-doped GaN layer. For this demonstration, only one broad-area mesa was defined per sample, $700 \mu \mathrm{m} \times 450 \mu \mathrm{m}$ in size, but the whole process could be applied to different mesa sizes, and would also work on mesa array. The PR mask remaining after mesa etching was washed off, and the samples underwent rapid thermal annealing at $500^{\circ} \mathrm{C}$ for $120 \mathrm{~s}$ in an oxygen-nitrogen ambient to form semi-transparent ohmic contacts to the p-GaN. Finally, n-pads and p-pads consisting of a $50 \mathrm{~nm} / 200 \mathrm{~nm} \mathrm{Ti} / \mathrm{Au}$ bilayer were deposited by sputtering and patterned by a lift-off process. In order to create the micro-image a new process step was added at the end of the standard process described above. This additional step consists of spin-coating a 500nm-thick PR (Microposit S1805) layer followed by standard photolithography to transfer the micro-image from a photo mask into the PR as shown in Fig. 1a. Subsequently the patterned samples underwent a room temperature plasma treatment in a reactive ion etch system with $15 \mathrm{sccm}$ of $\mathrm{CHF}_{3}$ and $5 \mathrm{sccm}$ of Ar. The plasma power and the chamber pressure were set at $200 \mathrm{~W}$ and $30 \mathrm{mTorr}$ respectively for a total treatment period of 120 s. The remaining PR mask was then washed off and the devices

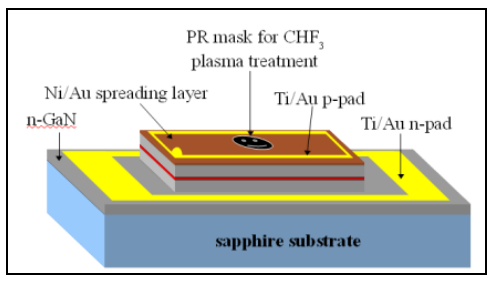

(a)

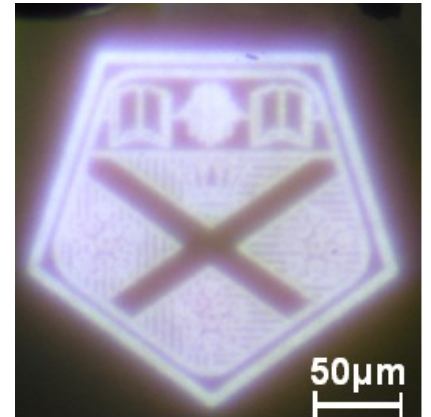

(b)

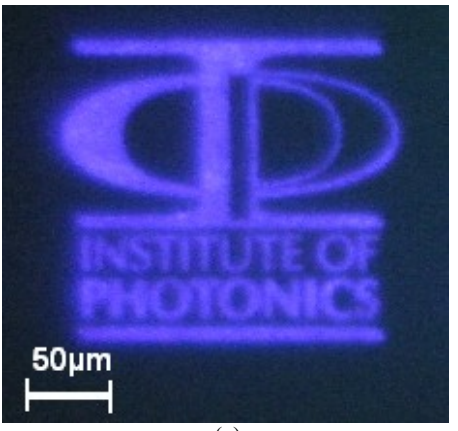

(c)

Figs. 1 - (a) Schematic structure of a LED with the patterned PR mask prior to the $\mathrm{CHF}_{3}$ plasma treatment and optical micrographs showing the logo of the University of Strathclyde (b) and the logo of the Institute of Photonics (c) displayed respectively by a $370 \mathrm{~nm}$ and a $450 \mathrm{~nm}$ patterned broad-area LED. 
were wire bonded onto printed circuit boards for characterisation.

Experimental results and discussion: Figs. $1 \mathrm{~b}$ and $1 \mathrm{c}$ show two turned-on devices corresponding to the two different emission wavelengths. Both pictures show high-fidelity display of micro-images, respectively, of the University of Strathclyde's logo (at 370nm) and of the Institute of Photonics' logo (at 450nm). We emphasise that these images are each emitted from a single LED device. The size of these images is typically $200 \mu \mathrm{m} \times 200 \mu \mathrm{m}$ and they show features as small as few microns demonstrating a resolution already much better than for a micro-LED-based display [3]. We note that the resolution achieved here is similar to the resolution of the UV photolithography used to define the PR pattern, and consequently we may expect better resolution with the use of higher resolution patterning methods such as e-beam lithography and nanoimprint lithography. Despite the selective reduction of the $\mathrm{p}-\mathrm{GaN}$ conductivity by the plasma treatment, the injection current versus the forward bias voltage (I-V) characteristics plotted in fig. 2.a show similar behaviour to standard untreated LEDs. We can deduce from this result that the PR-covered area of the p-GaN layer is unaffected by the $\mathrm{CHF}_{3}$ plasma treatment (meaning that the PR patterned layer acts as an efficient blocking layer), and also that the $\mathrm{Ni} / \mathrm{Au}$ spreading layer area not covered by the PR is undamaged during the plasma treatment. Indeed the emitting area is connected electrically to the p-pad only through the spreading layer covering the entire mesa surface as shown on Fig. 1.a. Therefore if the spreading layer was damaged either by any etching process or by any chemical reaction, the electrical contact would be dramatically degraded leading to no light emission or a very high differential resistance on the I-V characteristics, contrary to the observed device characteristics. While the mechanistic details are under ongoing study, we assume that the PR-uncovered $\mathrm{p}-\mathrm{GaN}$ area is passivated with the plasma treatment despite the top thin spreading layer, probably due to its high porosity after annealing. The nanoporous nature will enable the plasma species to easily diffuse through the thin metallic layer and then to interact with the underlying $\mathrm{p}-\mathrm{GaN}$ layer.

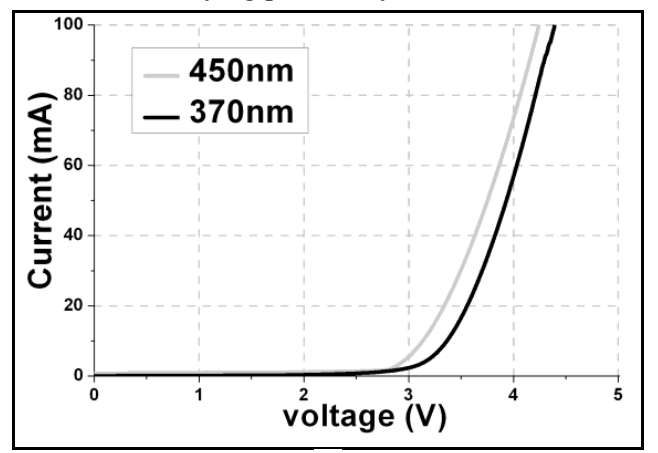

(a)

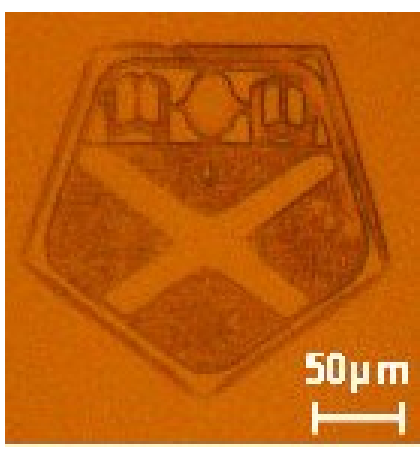

(b)

Figs. 2 - (a) I-V characteristics from the 370nm and 450nm patterned broad LEDs and (b) PR pattern made from the 1:1 imaging of the UV patterned image-containing LED.

In order to demonstrate the transfer of micro-images onto an application plane by projection, the $370 \mathrm{~nm}$ patterned LED was used for maskless photolithography by direct LED writing through a one-to-one imaging setup [5]. A silicon substrate with a $200 \mathrm{~nm}$-thick $\mathrm{SiO}_{2}$ top layer was covered by a $500 \mathrm{~nm}$-thick PR film (Microposit S1805) and placed underneath the projection optics. The photoresist soft-bake, exposure dose and development time were obtained from the manufacturer's datasheet. The UV LED was driven at an injection current of $20 \mathrm{~mA}$, giving a projected optical power density of about $12.5 \mathrm{~W} . \mathrm{cm}^{-2}$ at the focal plane. The exposure dose was controlled by a shutter placed directly in front of the collection optics. A good transfer of the microimage was achieved in this demonstration as shown in Fig. 2b. We anticipate that better resolution of the transferred image will be obtained with further optimisation of the imaging setup and LED writing parameters.

Conclusions: In summary, we show that selective lateral modulation of the conductivity of $\mathrm{p}-\mathrm{GaN}$ by applying a specific plasma treatment through a patterned photoresist mask allows fabrication of novel imagecontaining LEDs. These images can be displayed as fixed patterns, or projected into a variety of materials and application planes for colour conversion and scientific and instrumentation applications.

\section{References:}

1 - I. Ozden et al., Phys. Stat. Sol. (a), vol. 188, no. 1, pp. 139-142, Nov. 2001.

2 - E. F. Schubert and J. K. Kim, Science, vol. 308. pp. 1274-1278, May 2005.

3 - Z. Gong et al., IEEE Trans. Electron Devices, vol. 54, no. 10, pp. 2650-2658, Oct. 2007.

4 - D. Massoubre et al., LEOS 2009, MK2, Belek-Antalya, Turkey, Oct. 2009.

5 - D. Elfström et al., Optics Express, Vol. 17, no. 26, pp. 23522-23529, Dec. 2009. 